\title{
QCD at small $x$ : From Color Glass Condensate to Pomerons and Odderons and more
}

\author{
Jamal Jalilian-Marian ${ }^{1,2, a}$ \\ ${ }^{1}$ Department of Natural Sciences, Baruch College, New York NY 10010 \\ ${ }^{2}$ CUNY Graduate Center, New York NY 10016
}

\begin{abstract}
We show that JIMWLK evolution equation [1] for quadrupole, normalized trace of 4 Wilson lines, reduces to BJKP equation [2] for the exchange of 4-Reggeized gluons in the dilute region (weak field limit). We comment on how this may be generalized to the exchange of arbitrary number of Reggeized gluons and how one may derive expressions for multi-pomeron vertices.
\end{abstract}

\section{Introduction}

Gluon saturation is expected to pay a dominant role in high energy processes at low to intermediate momentum transfer [3]. An effective theory of QCD at high energy which incorporates saturation dynamics is referred to as Color Glass Condensate (CGC) formalism [1]. The CGC formalism re-sums coherent multiple scatterings as well as a resummation of large energy logs that appear in physical cross sections when center of mass energy of a collision becomes large. It has been successfully applied to many processes in high energy collisions where at least one of the participants is a hadron/nucleus. In most physical processes described by the CGC formalism, the basic ingredient is the dipole cross section, trace of two Wilson lines, each describing eikonal propagation of a quark (anti-quark) in the background of the target color field [4]. A recent measurement of di-hadron azimuthal angular correlations in proton (deuteron)-nucleus collisions [5, 6] has generated much interest in the degrees of freedom in the CGC formalism since di-hadron correlations involve trace of 4 Wilson lines, known as a quadrupole. Important progress has been made in understanding the evolution equation the quadrupole satisfies [7], as well as generic properties of its solution [8]. Here we focus on the perturbative limit of the quadrupole evolution equation and show that it reduces to the known BJKP equation. We then outline how one may show this correspondence in the general case of $n$-Reggeized exchange as well as deriving an expression for the $m \rightarrow n$ pomeron vertex.

\section{Evolution equation}

The quadrupole is defined as

$$
Q(r, \bar{r}, \bar{s}, s) \equiv \frac{1}{N_{c}} \operatorname{Tr} V_{r} V_{\bar{r}}^{\dagger} V_{\bar{s}} V_{s}^{\dagger}
$$

\footnotetext{
a e-mail: jamal.jalilian-marian@baruch.cuny.edu
} 
where $V(r)$ is a Wilson line in the fundamental representation of $S U\left(N_{c}\right)$ gauge group and $r, \bar{r}, s, \bar{s}$ are two-dimensional transverse coordinates. $V(r)$ is defined as

$$
V\left(r_{t}\right) \equiv \hat{P} e^{-i g \int d x^{-} A^{+}}
$$

where $A_{a}^{\mu}\left(x^{-}, r_{t}\right)=\delta^{\mu+} \delta\left(x^{-}\right) \alpha_{a}\left(r_{t}\right)$ is the gluon field in the covariant gauge and is related to the color charge density by $\partial_{t}^{2} \alpha^{a}\left(r_{t}\right) \sim g \rho^{a}\left(r_{t}\right)$.

The JIMWLK evolution equation for the expectation value of any operator $\langle O\rangle$ is

$$
\frac{d}{d y}\langle O\rangle=\frac{1}{2}\left\langle\int d^{2} x d^{2} y \frac{\delta}{\delta \alpha_{x}^{b}} \eta_{x y}^{b d} \frac{\delta}{\delta \alpha_{y}^{d}} O\right\rangle
$$

with

$$
\eta_{x y}^{b d}=\frac{1}{\pi} \int \frac{d^{2} z}{(2 \pi)^{2}} \frac{(x-z) \cdot(y-z)}{(x-z)^{2}(y-z)^{2}}\left[1+U_{x}^{\dagger} U_{y}-U_{x}^{\dagger} U_{z}-U_{z}^{\dagger} U_{y}\right]^{b d}
$$

where $U$ is the analog of eq. (2) in the adjoint representation. Applying this to the quadrupole operator given by eq. (1) gives

$$
\begin{aligned}
& \frac{d}{d y}\langle Q(r, \bar{r}, \bar{s}, s)\rangle= \\
& \left.\frac{N_{c} \alpha_{s}}{(2 \pi)^{2}} \int d^{2} z\right\}\left\langle\left[\frac{(r-\bar{r})^{2}}{(r-z)^{2}(\bar{r}-z)^{2}}+\frac{(r-s)^{2}}{(r-z)^{2}(s-z)^{2}}-\frac{(\bar{r}-s)^{2}}{(\bar{r}-z)^{2}(s-z)^{2}}\right] Q(z, \bar{r}, \bar{s}, s) S(r, z)+\right. \\
& {\left[\frac{(r-\bar{r})^{2}}{(r-z)^{2}(\bar{r}-z)^{2}}+\frac{(\bar{r}-\bar{s})^{2}}{(\bar{r}-z)^{2}(\bar{s}-z)^{2}}-\frac{(r-\bar{s})^{2}}{(r-z)^{2}(\bar{s}-z)^{2}}\right] Q(r, z, \bar{s}, s) S(z, \bar{r})+} \\
& {\left[\frac{(\bar{r}-\bar{s})^{2}}{(\bar{r}-z)^{2}(\bar{s}-z)^{2}}+\frac{(s-\bar{s})^{2}}{(s-z)^{2}(\bar{s}-z)^{2}}-\frac{(\bar{r}-s)^{2}}{(s-z)^{2}(\bar{r}-z)^{2}}\right] Q(r, \bar{r}, z, s) S(\bar{s}, z)+} \\
& {\left[\frac{(r-s)^{2}}{(r-z)^{2}(s-z)^{2}}+\frac{(s-\bar{s})^{2}}{(s-z)^{2}(\bar{s}-z)^{2}}-\frac{(r-\bar{s})^{2}}{(r-z)^{2}(\bar{s}-z)^{2}}\right] Q(r, \bar{r}, \bar{s}, z) S(z, s)-} \\
& {\left[\frac{(r-\bar{r})^{2}}{(r-z)^{2}(\bar{r}-z)^{2}}+\frac{(s-\bar{s})^{2}}{(s-z)^{2}(\bar{s}-z)^{2}}+\frac{(r-s)^{2}}{(r-z)^{2}(s-z)^{2}}+\frac{(\bar{r}-\bar{s})^{2}}{(\bar{r}-z)^{2}(\bar{s}-z)^{2}}\right] Q(r, \bar{r}, \bar{s}, s)-} \\
& {\left[\frac{(r-s)^{2}}{(r-z)^{2}(s-z)^{2}}+\frac{(\bar{r}-\bar{s})^{2}}{(\bar{r}-z)^{2}(\bar{s}-z)^{2}}-\frac{(\bar{r}-s)^{2}}{(\bar{r}-z)^{2}(s-z)^{2}}-\frac{(r-\bar{s})^{2}}{(r-z)^{2}(\bar{s}-z)^{2}}\right] S(r, s) S(\bar{r}, \bar{s})-} \\
& \left.\left.\left[\frac{(r-\bar{r})^{2}}{(r-z)^{2}(\bar{r}-z)^{2}}+\frac{(s-\bar{s})^{2}}{(s-z)^{2}(\bar{s}-z)^{2}}-\frac{(r-\bar{s})^{2}}{(r-z)^{2}(\bar{s}-z)^{2}}-\frac{(\bar{r}-s)^{2}}{(\bar{r}-z)^{2}(s-z)^{2}}\right] S(r, \bar{r}) S(\bar{s}, s)\right]\right\}
\end{aligned}
$$

and we have defined the $S$ matrix as

$$
S(r, \bar{r}) \equiv \frac{1}{N_{c}} \operatorname{Tr} V_{r} V_{\bar{r}}^{\dagger}
$$

Since we are interested in the dilute region, we need to expand the Wilson lines in the evolution equation above. Here it is easier to work with the so called $T_{Q}=1-Q, T=1-S$ matrices rather than $Q$ and $S$, we refer the reader to [7] for details. Keeping the linear terms in the expansion of the Wilson lines, the $O\left(\alpha^{2}\right)$ terms give

$$
T_{Q}(r, \bar{r}, \bar{s}, s) \rightarrow T(r, \bar{r})+T(\bar{s}, s)-T(r, \bar{s})-T(\bar{r}, s)+T(r, s)+T(\bar{r}, \bar{s})
$$


such that each $T$ of a given argument satisfies the BFKL equation, for example,

$$
\frac{d}{d y}\langle T(r, s)\rangle=\frac{N_{c} \alpha_{s}}{2 \pi^{2}} \int d^{2} z \frac{(r-s)^{2}}{(r-z)^{2}(s-z)^{2}}[\langle T(r, z)\rangle+\langle T(z, s)\rangle-\langle T(r, s)\rangle]
$$

where $T$ here stands for

$$
T(r, \bar{r}) \rightarrow \Gamma(r-\bar{r}) \sim g^{2} \alpha^{a}(r) \alpha^{a}(\bar{r}) .
$$

This means that one should have back to back correlations in di-hadron production when the transverse momenta of the produced hadrons are large (hence the expansion in the gluon field). Going beyond BFKL requires that we look at terms of $O\left(\alpha^{4}\right)$. Again we expand each Wilson line, and Fourier transform to momentum space to get

$$
\begin{aligned}
\frac{d}{d y} \hat{T}_{4}\left(l_{1}, l_{2}, l_{3}, l_{4}\right) & =\frac{N_{c} \alpha_{s}}{\pi^{2}} \int d^{2} p_{t}\left[\frac{p^{i}}{p_{t}^{2}}-\frac{\left(p^{i}-l_{1}^{i}\right)}{\left(p_{t}+l_{1}\right)^{2}}\right] \cdot\left[\frac{p^{i}}{p_{t}^{2}}-\frac{\left(p^{i}-l_{2}^{i}\right)}{\left(p_{t}+l_{2}\right)^{2}}\right] \hat{T}_{4}\left(p_{t}+l_{1}, l_{2}-p_{t}, l_{3}, l_{4}\right) \\
& +\cdots \\
& -\frac{N_{c} \alpha_{s}}{(2 \pi)^{2}} \int d^{2} p_{t}\left[\frac{l_{1}^{2}}{p_{t}^{2}\left(l_{1}-p_{t}\right)^{2}}+\left\{l_{1} \rightarrow l_{2}, l_{3}, l_{4}\right\}\right] \hat{T}_{4}\left(l_{1}, l_{2}, l_{3}, l_{4}\right)
\end{aligned}
$$

where $\hat{T}_{4}$ is defined as $\hat{T}_{4}\left(l_{1}, l_{2}, l_{3}, l_{4}\right)=\frac{1}{N_{c}} \operatorname{Tr} \rho\left(l_{1}\right) \rho\left(l_{2}\right) \rho\left(l_{3}\right) \rho\left(l_{4}\right)$. Eq. (10) is identical to the BJKP equation [2] for 4-Reggeized gluon exchange state. This contribution corresponds to double scatterings in di-hadron production cross section and is responsible for reducing the angular correlations as one lowers the away side hadron's transverse momentum (we note that photon-hadron angular correlations involve dipoles only [9]).

Going beyond 4-Reggeized gluons is straight forward but Algebraically tedious. It would correspond to progressively including higher number of re-scattering in the cross section. One needs to write the JIMWLK equation for the evolution of $n$ Wilson lines, expand each one to the lowest nontrivial order in the gauge field and Fourier transform, this is work in progress and will be reported elsewhere. Following the same approach one can derive expressions for $m \rightarrow n$ pomeron vertices (see [10] for a derivation of triple pomeron vertex) .

\section{References}

[1] J. Jalilian-Marian, A. Kovner, L. D. McLerran and H. Weigert, Phys. Rev. D 55, 5414 (1997); J. Jalilian-Marian, A. Kovner, A. Leonidov and H. Weigert, Nucl. Phys. B 504, 415 (1997), Phys. Rev. D 59, 014014 (1999), Phys. Rev. D 59, 014015 (1999), Phys. Rev. D 59, 034007 (1999), A. Kovner, J. G. Milhano and H. Weigert, Phys. Rev. D 62, 114005 (2000); A. Kovner and J. G. Milhano, Phys. Rev. D 61, 014012 (2000); E. Iancu, A. Leonidov and L. D. McLerran, Nucl. Phys. A 692, 583 (2001), Phys. Lett. B 510, 133 (2001); E. Ferreiro, E. Iancu, A. Leonidov and L. McLerran, Nucl. Phys. A 703, 489 (2002); H. Weigert, Nucl. Phys. A703, 823-860 (2002). J. -P. Blaizot, E. Iancu, H. Weigert, Nucl. Phys. A713, 441-469 (2003); J. Jalilian-Marian, S. Jeon and R. Venugopalan, Phys. Rev. D 63, 036004 (2001) [hep-ph/0003070].

[2] J. Bartels, Nucl. Phys. B 175, 365 (1980); T. Jaroszewicz, Acta Phys. Polon. B 11, 965 (1980); J. Kwiecinski and M. Praszalowicz, Phys. Lett. B 94, 413 (1980); Z. Chen, A. H. Mueller, Nucl. Phys. B451, 579-604 (1995).

[3] N. Armesto, N. Borghini, S. Jeon, U. A. Wiedemann, S. Abreu, V. Akkelin, J. Alam and J. L. Albacete et al., J. Phys. G 35, 054001 (2008) [arXiv:0711.0974 [hep-ph]]; F. Gelis, E. Iancu, J. Jalilian-Marian, R. Venugopalan, Ann. Rev. Nucl. Part. Sci. 60, 463-489 (2010); J. JalilianMarian, Y. V. Kovchegov, Prog. Part. Nucl. Phys. 56, 104-231 (2006). 
[4] A. Dumitru and J. Jalilian-Marian, Phys. Rev. Lett. 89, 022301 (2002) [hep-ph/0204028]; A. Dumitru, A. Hayashigaki and J. Jalilian-Marian, Nucl. Phys. A 765, 464 (2006) [hep-ph/0506308]; J. Jalilian-Marian and A. H. Rezaeian, Phys. Rev. D 85, 014017 (2012) [arXiv:1110.2810 [hep$\mathrm{ph}]$.

[5] E. Braidot [ STAR Collaboration ], Nucl. Phys. A854, 168-174 (2011); A. Adare et al. [PHENIX Collaboration], Phys. Rev. Lett. 107, 172301 (2011).

[6] C. Marquet, Nucl. Phys. A 796, 41 (2007); K. Tuchin, Nucl. Phys. A 846, 83 (2010); J. L. Albacete and C. Marquet, Phys. Rev. Lett. 105, 162301 (2010); D. Kharzeev, E. Levin and L. McLerran, Nucl. Phys. A 748, 627 (2005); A. Kovner, M. Lublinsky, Phys. Rev. D83, 034017 (2011); Phys. Rev. D 84, 094011 (2011); A. Stasto, B. -W. Xiao, F. Yuan, Phys. Lett. B 716, 430 (2012).

[7] J. Jalilian-Marian and Y. V. Kovchegov, Phys. Rev. D 70, 114017 (2004) [Erratum-ibid. D 71; F. Dominguez, A. H. Mueller, S. Munier and B. -W. Xiao, Phys. Lett. B 705, 106 (2011); J. JalilianMarian, Phys. Rev. D 85, 014037 (2012); A. Dumitru and J. Jalilian-Marian, Phys. Rev. D 81, 094015 (2010); A. Dumitru, J. Jalilian-Marian, Phys. Rev. D82, 074023 (2010). E. Iancu, D. N. Triantafyllopoulos, JHEP 1111, 105 (2011).

[8] A. Dumitru, J. Jalilian-Marian, T. Lappi, B. Schenke, R. Venugopalan, Phys. Lett. B 706, 219 (2011); A. Dumitru, J. Jalilian-Marian and E. Petreska, Phys. Rev. D 84, 014018 (2011) [arXiv:1105.4155 [hep-ph]].

[9] J. Jalilian-Marian and A. H. Rezaeian, Phys. Rev. D 86, 034016 (2012); J. Jalilian-Marian, Eur. Phys. J. C 61, 789 (2009), Nucl. Phys. A 770, 210 (2006), Nucl. Phys. A 739, 319 (2004) [nuclth/0402014], Phys. Rev. C 70, 027902 (2004) [nucl-th/0212018].

[10] G. A. Chirilli, L. Szymanowski and S. Wallon, Phys. Rev. D 83, 014020 (2011) [arXiv:1010.0285 [hep-ph]]. 